\title{
Benchmarking the Science for the Southern Wide-Field Gamma-ray Observatory (SWGO)
}

\section{Ulisses Barres de Almeida, ${ }^{a, *}$ Gwenael Giacinti $^{b}$ and Francesco Longo ${ }^{c, d}$ on behalf of the SWGO Collaboration}

(a complete list of authors can be found at the end of the proceedings)

${ }^{a}$ Centro Brasileiro de Pesquisas Físicas (CBPF),

Rua Dr. Xavier Sigaud 150, 22290-180 Rio de Janeiro, Brazil

${ }^{b}$ Max-Planck-Institut für Kernphysik,

Saupfercheckweg 1, 69117 Heidelberg, Germany

${ }^{c}$ Università degli Studi di Trieste, Dipartimento di Fisica

via A. Valerio 2, 34127 Trieste, Italy

${ }^{d}$ INFN - Sezione di Trieste,

via A. Valerio 2, 34127 Trieste, Italy

E-mail: ulisses@cbpf.br, swgo_spokespersons@swgo.org

The Southern Wide-field Gamma-ray Observatory (SWGO) is the project to build a new extensive air shower particle detector for the observation of very-high-energy gamma-rays in South America. SWGO is currently planned for installation in the Southern Hemisphere, which grants it a unique science potential among ground-based gamma-ray detectors. It will complement the capabilities of CTA, working as a wide-field instrument for the monitoring of transient and variable phenomena, and will expand the sky coverage of Northern Hemisphere facilities like HAWC and LHAASO, thus granting access to the entire Galactic Plane and the Galactic Center. SWGO aims to achieve excellent sensitivity over a very large target energy range from about $100 \mathrm{GeV}$ to the $\mathrm{PeV}$, and improve on the performance of current sampling array instruments in all observational parameters, including energy and angular resolution, background rejection, and single-muon detection capabilities. The directives for the final observatory design will be given by a number of key science goals which are being defined over the course of the Project's R\&D phase. In this contribution we will present the core science topics and target performance goals that serve as benchmarks to guide SWGO's design configuration.

$37^{\text {th }}$ International Cosmic Ray Conference (ICRC 2021)

July 12th - 23rd, 2021

Online - Berlin, Germany

\footnotetext{
*Presenter
} 


\section{Introduction}

The direct detection of primary gamma-rays is only possible with space-based detectors, such as Fermi-LAT. However, the size and sensitivity limitations of satellite-borne detectors are prohibitive factors as fluxes become small towards higher energies. An alternative solution is to use the shower of particles produced as gamma-rays interact with the atmosphere for an indirect detection. These showers can be studied with observatories of two complementary types: imaging atmospheric Cherenkov telescopes, which are narrow field-of-view, highly sensitive pointing instruments, such as CTA [16], and high altitude air shower arrays, such as SWGO. Wide-field air shower arrays have the highest energy reach, and are ideal to search for transient sources (monitoring), and for the study of emission from very extended regions of the sky (surveying).

The potential of such a wide-field, and high duty-cycle, ground-based gamma-ray detector to address this observational programme has been demonstrated by the current-generation instruments HAWC [24] and ARGO [21], and has been recently extended in the Northern Hemisphere by LHAASO [8]. Nevertheless, no such instrument exists in the south, where there is great potential for the mapping of large scale Galactic emission as well as providing access to the full sky for transient and variable phenomena, including multi-messenger events [5]. Access to the Galactic Centre and complementarity with the major facility CTA-South are key motivations for the installation of such an observatory. There is also significant potential for cosmic-ray studies, including anisotropy, where the tropical latitude range between $10^{\circ}$ and $30^{\circ}$ represents a visibility gap for joint analysis between northern facilities and IceCube, at the South Pole [13].

\subsection{The SWGO concept}

The SWGO aims to cover an extended energy range, from the low energies, around 100 $\mathrm{GeV}$, thus closing the gap between wide-field satellite and ground-based measurements, to the very-high energies, at the $\mathrm{PeV}$ scale. At low energies, the critical design factors are the amount of signal collected and the ability to cope with explosive trigger rates, whereas gamma/hadron discrimination will be based on the comparison of shower patterns at ground, exploiting the PMTs signal time resolution, e.g. [12]. At higher energies, the density of stations needed to ensure a good gamma/hadron discrimination based on individual muon tagging, and improved energy resolution, are crucial requirements [10], constraining the capacity to cover large areas at a limited cost.

Given those considerations, the general design concept of SWGO [14], which will serve as a baseline for further studies in the current $R \& D$ phase of the project, consists in a large (circa 80,000 $\left.\mathrm{m}^{2}\right)$ and high fill-factor ( $\sim 80 \%$, corresponding to $4-5 \times$ the HAWC effective area) core array of water Cherenkov detector (WCD) units, surrounded by an outrigger array of WCDs, arranged in a low fill-factor grid and covering an area of minimum 200,000 $\mathrm{m}^{2}$ to provide high-energy sensitivity (but possibly extending up to the $\mathrm{km}^{2}$ scale to match LHAASO's UHE capabilities). The array is to be deployed at a high-altitude site in the Andes, at least $4.4 \mathrm{~km}$ above sea level [11].

The WCD detector units are optically isolated water volumes instrumented with photo-detectors and able to sample the shower front to provide time and particle energy density information, as well as muon tagging for background rejection. The final detector choices - if the WCD units will consist of tanks, bags deployed in a lake, or assembled in a pond-like structure - are still under investigation, as well as details of the individual detector unit design, photosensors and electronics. 


\section{The SWGO Science Case}

SWGO will probe extreme phenomena and astrophysical environments to address some of the most compelling questions of astro-particle and high-energy astrophysics of today, such as the origin of $\mathrm{PeV}$ cosmic rays and the nature of dark matter particles, as well as the study of highly energetic transient phenomena [5]. For many of its scientific objectives, the SWGO concept occupies a niche, which in some cases is unrivalled by current or planned facilities, given, among other reasons, the observatory's southern location, which will allow for direct observation of the Galactic Centre and the 'Fermi Bubbles', with ample view of the Galactic Plane. The new observatory will be a powerful time-variability monitor, mainly due to its planned lower-energy threshold, filling an empty place in the global multi-messenger network of gravitational, electromagnetic and neutrino observatories. In this regard, it should be able to issue alerts and be fully complementary to the next-generation Cherenkov Telescope Array, the CTA.

In the following, we will briefly describe the core science goals of SWGO, and their principal associated design characteristics, which together form a basis of justification for the design work of the current R\&D phase of the project. Here, the current results from HAWC, and more recently LHAASO, provide a baseline for the new observatory science objectives, for which a detailed description was presented in an earlier white-paper by the Collaboration [5].

\subsection{PeVatrons and galactic accelerators}

At the high extreme of SWGO's energy range, science is dominated by the search for PeVatrons. These are the putative sources responsible for the acceleration of knee cosmic-ray particles, whose signature in gamma rays is expected to come in the form of a hard spectrum, with emission unabated beyond $100 \mathrm{TeV}$ [7]. This science case has recently been reinforced by a detection, by the Tibet AS+MD array, of diffuse gamma-ray emission in the Galaxy well above this energy range [6], and by LHAASO's detection of a number of PeV-emitting gamma-ray sources in the Galactic Plane [27]. The identification of PeVatrons by means of the detection of a high-energy spectral cutoff signature (or lack thereof) poses a requirement on the energy resolution of the observatory, of $\sim 30 \%$, in the energy range above $100 \mathrm{TeV}$. A firm estimate on the required point-source sensitivity at these energies depends on knowledge of the Galactic hard-spectrum sources, which is largely lacking, but recent estimates from [7] suggest that an integral sensitivity above $100 \mathrm{TeV}$ at the $10^{-13} \mathrm{ph} \cdot \mathrm{cm}^{-2} \cdot \mathrm{s}^{-1}$ level (for 1-year integration) is required for an in-depth probe of the Galactic PeVatron population, an estimate needing further consideration in face of the most recent LHAASO results [27].

The study of extended sources and of diffuse emission, whose highest-energy flux is generally linked to the fraction of PeVatrons among the Galactic accelerators, will critically depend on the background rejection power of the array, which aims to achieve a residual charged cosmic-ray background better than $10^{-3}$ at $10 \mathrm{TeV}$, and beyond $\sim 10^{-4}$ above $100 \mathrm{TeV}$. Above $\sim 10 \mathrm{TeV}, \gamma /$ hadron separation is strongly associated with the capability to detect single muons at the individual detector units. The science case for extended sources will be centred on the study of PWNe and TeV Halos, which pose the principal benchmark constraints for the angular resolution of the observatory. Here, the goal will be to extend the detectability of these objects towards higher energies, above $10 \mathrm{TeV}$, and for angular extensions up to $\sim 3^{\circ}$, for which a relatively good angular resolution capability of circa $0.15^{\circ}$ is estimated to be necessary to resolve the vast majority (over $97 \%$ ) of the population [19]. 

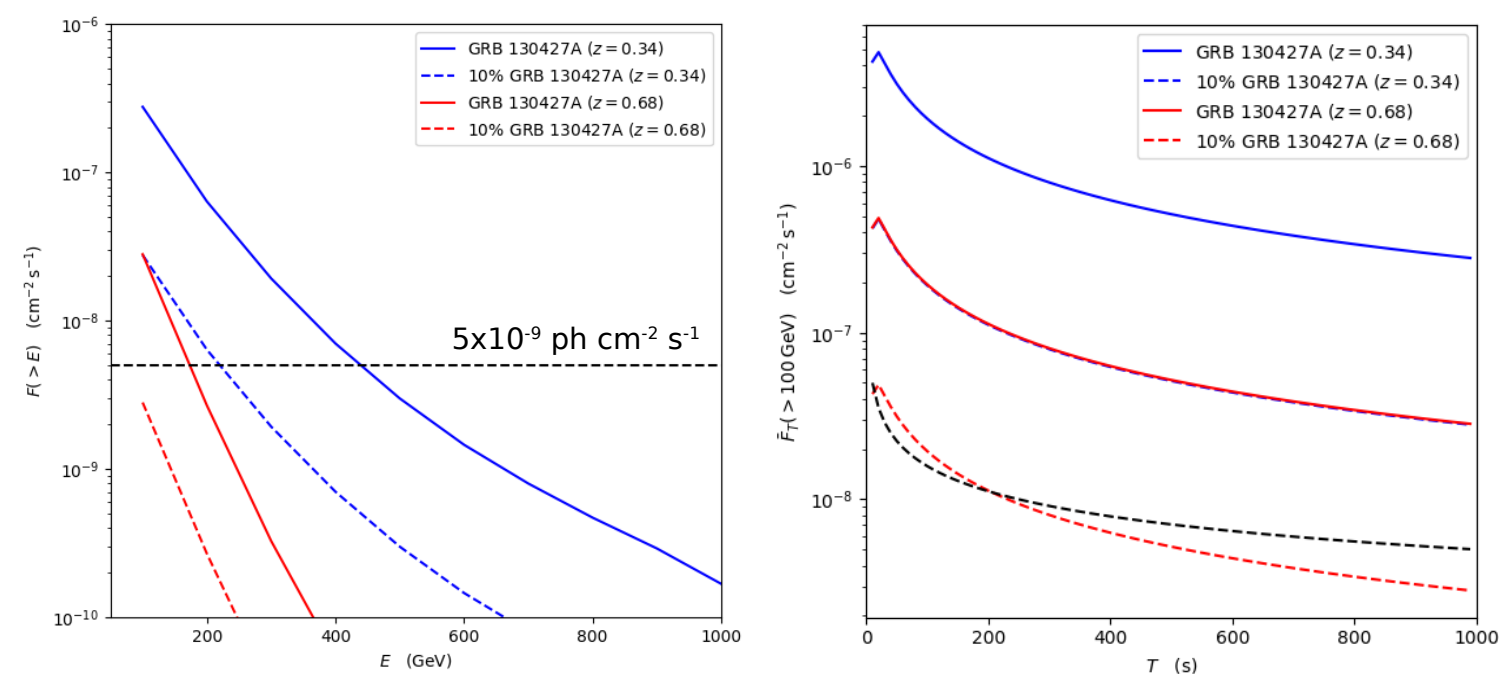

Figure 1: Expected photon flux at VHE energies from a light-curve parameterization [18] of the bright GRB 130427A as seen by Fermi-LAT [3]. The black dashed line indicates a target integral sensitivity for SWGO between 0.1 and $1 \mathrm{TeV}$ for $1 \mathrm{ks}$ integration time. The left plot shows the detectability, for various scalings of the integral source flux, as a function of energy, indicating the importance of a low-energy detection threshold for the experiment. The right plot presents the integral source flux above $100 \mathrm{GeV}$ as a function of time since $t_{0}$, showing that an instrument like SWGO could perform well in detecting early-time triggers from GRBs.

\subsection{Gamma-ray Bursts and transients}

At the lowest energies, the core science of SWGO is focused on transient sources, exploiting the wide-field of view and near-continuous duty cycle of the observatory, along with its southern location, to work as a monitoring and trigger instrument complementary to CTA. At the few-100 GeV energy scale, the two principal target sources are Active Galactic Nuclei (AGN) and GammaRay Bursts (GRB), both of which are the candidate multi-messenger astrophysical counterparts of very high energy (VHE) neutrinos and gravitational wave (GW) events, respectively [1, 2, 15]. In terms of performance goals, the idea in the transients science case is to guarantee that SWGO will be an effective detector of serendipitous variable phenomena, able to efficiently trigger other instruments within short timescales, particularly in the case of GRBs where it should have good performance in the first few kiloseconds from $t_{0}$, when source flux is higher [4].

Figure 1 presents an analysis of the detectability of the bright GRB $130427 \mathrm{~A}^{1}$ at VHE energies, based on a light-curve extrapolation into the VHE energies [18]. The calculations show that a target integral sensitivity $\sim 10^{-9} \mathrm{ph} . \mathrm{cm}^{-2} \cdot \mathrm{s}^{-1}$ between $0.1-1 \mathrm{TeV}$ (in $1 \mathrm{ks}$ ) is able to detect a source only a fraction of the brightness of GRB 130427A. This level roughly corresponds to the $1 / 6$ brightest GRBs in the Fermi-LAT catalogue, and is statistically equivalent to securing one safe serendipitous GRB detection by SWGO per year, and should also be sufficient to guarantee regular (monthly) AGN flare triggers [17]. For bright, and relatively nearby GRBs, such as GRB 190114C [20], this sensitivity would allow not only for the generation of an early $(<1 \mathrm{ks})$ trigger alert by SWGO, but also, in case of favourable-condition observations, of a short-time resolution of the early emission at or below the $100 \mathrm{~s}$ timescale [18], with the possibility of probing the prompt phase at VHEs.

\footnotetext{
${ }^{1}$ From which Fermi-LAT detected its highest-energy photon of $94 \mathrm{GeV}$ [3]
} 

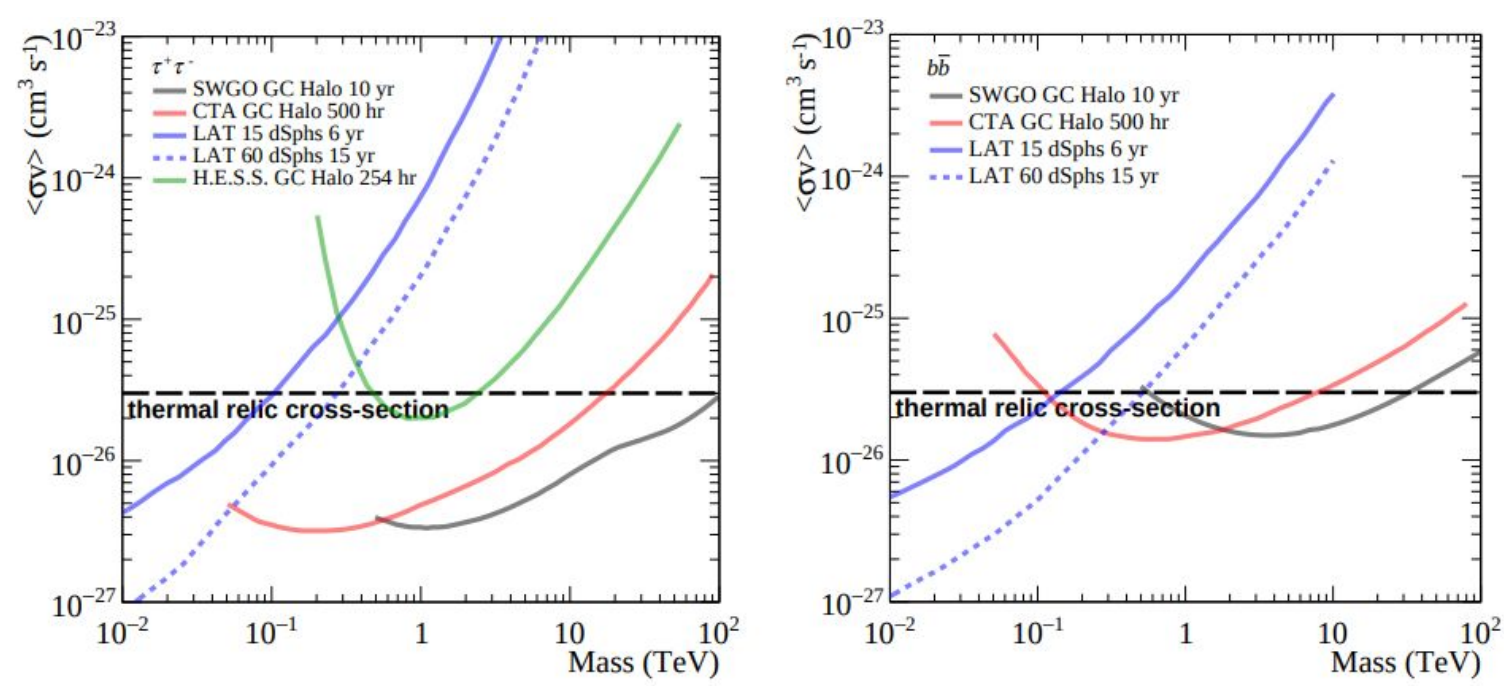

Figure 2: Expected sensitivity of SWGO to the detection of thermal relic Dark Matter signals from the Galactic Center Halo for the channels $\tau^{+} \tau^{-}$and $b \bar{b}$ [25]. The plots shows that the expected sensitivity of SWGO should allow probing deep into the entire range of the thermal relic cross-section limits, extending CTA capabilities above a few $\mathrm{TeV}$, up to $100 \mathrm{TeV}$.

\subsection{Dark Matter searches}

The sensitivity and energy resolutions of SWGO should also be sufficient to constrain the science goals for Dark Matter observations, for which the Galactic Centre Halo is the most promising observational target, along with some potentially interesting ultra-faint Dwarf Spheroidal galaxies (DSphs) that could be detected e.g., by LSST [23]. As shown in Figure 2, the niche of SWGO in the study of Dark Matter is the possibility to constrain the entire energy range of WIMP models, especially above $30 \mathrm{TeV}$, where SWGO sensitivity could outperform that of CTA for reasonable integration times. For that, a point-source flux sensitivity of $\approx 3 \times 10^{-14} \mathrm{erg} . \mathrm{cm}^{-2} \cdot \mathrm{s}^{-1}$ in the range between a few to several $10 \mathrm{~s} \mathrm{TeV}$ (for 5-year integration), as used in the figure, is the target goal.

\subsection{Cosmic-ray studies}

As already mentioned, at high energies, generally above $\sim 10 \mathrm{TeV}$, the capability to detect single muons is crucial for a good gamma-hadron separation, and this capability would impact not only the background rejection necessary for improving detectability of faint diffuse gamma-ray emission (as discussed in [22]), but would also allow for unique mass-resolved charged cosmic-ray studies up to $\mathrm{PeV}$ energies. The science goal here is ultimately to understand the nature of the evolution of the cosmic-ray dipole and multipoles between $0.1-$ multi PeV, and is subdivided into:

- extending the dipole anisotropy detection to beyond the $1 \mathrm{PeV}$ energy (at $10^{-3}$ level);

- achieving the decomposition of the cosmic ray spectrum into four mass groups $A=\{1,4,14$, $56\}$, in the $0.1-1 \mathrm{PeV}$ energy range;

- measuring the multipole $l$ - scale of the cosmic-ray anisotropy, for events with energies above $0.1 \mathrm{PeV}$, and in particular extending it beyond $l=5\left(\sim 35^{\circ}\right)$ above $0.1 \mathrm{PeV}$, and beyond $l=20$ $\left(\sim 10^{\circ}\right)$ around $10 \mathrm{TeV}$ (at $10^{-3}$ level). 


\begin{tabular}{|c|c|c|}
\hline Science Case & Design Drivers & Benchmark Description \\
\hline $\begin{array}{l}\text { Transient Sources: } \\
\text { Gamma-ray Bursts }\end{array}$ & $\begin{array}{l}\text { Low-energy sensitivity \& } \\
\text { Site altitude }{ }^{a}\end{array}$ & $\begin{array}{l}\text { Min. time for } 5 \sigma \text { detection: } \\
\mathrm{F}(100 \mathrm{GeV})=10^{-8} \mathrm{erg} / \mathrm{cm}^{2} . \mathrm{s} \\
\mathrm{PWL} \text { index }=-2 ., \mathrm{F}(\mathrm{t}) \propto t^{-1.2}\end{array}$ \\
\hline $\begin{array}{l}\text { Galactic Accelerators: } \\
\text { PeVatron Sources }\end{array}$ & $\begin{array}{l}\text { High-energy sensitivity \& } \\
\text { Energy resolution }^{b}\end{array}$ & $\begin{array}{l}\text { Maximum exp-cutoff energy de- } \\
\text { tectable } 95 \% \mathrm{CL} \text { in } 5 \text { years for: } \\
\mathrm{F}(1 \mathrm{TeV})=5 \mathrm{mCrab} \text {, index }=-2.3\end{array}$ \\
\hline $\begin{array}{l}\text { Galactic Accelerators: } \\
\text { PWNe and TeV Halos }\end{array}$ & $\begin{array}{l}\text { Extended source sensitivity } \\
\& \text { Angular resolution }{ }^{c}\end{array}$ & $\begin{array}{l}\text { Max. angular extension detected } \\
\text { at } 5 \sigma \text { in } 5 \text {-yr integration for: } \\
\mathrm{F}(>1 \mathrm{TeV})=5 \times 10^{-13} \mathrm{TeV} / \mathrm{cm}^{-2} . \mathrm{s}\end{array}$ \\
\hline $\begin{array}{l}\text { Diffuse Emission: } \\
\text { Fermi Bubbles }\end{array}$ & Background rejection & $\begin{array}{l}\text { Minimum diffuse cosmic-ray } \\
\text { residual background level. } \\
\text { Threshold: }<10^{-4} \text { level at } 1 \mathrm{TeV} \text {. }\end{array}$ \\
\hline $\begin{array}{l}\text { Fundamental Physics: } \\
\text { Dark Matter from GC Halo }\end{array}$ & $\begin{array}{l}\text { Mid-range energy sensitivity } \\
\text { Site latitude }^{d}\end{array}$ & $\begin{array}{l}\text { Max. energy for } b \bar{b} \text { thermal relic } \\
\text { cross-section limit at 95\% CL in } \\
\text { 5-years, for Einasto profile. }\end{array}$ \\
\hline $\begin{array}{l}\text { Cosmic-rays: } \\
\text { Mass-resolved dipole / } \\
\text { multipole anisotropy }\end{array}$ & Muon counting capability ${ }^{e}$ & $\begin{array}{l}\text { Max. dipole energy at } 10^{-3} \text { level; } \\
\text { Log-mass resolution at } 1 \mathrm{PeV}- \\
\text { goal is } \mathrm{A}=\{1,4,14,56\} ; \text { Maxi- } \\
\text { mum multipole scale }>0.1 \mathrm{PeV}\end{array}$ \\
\hline
\end{tabular}

Table 1: SWGO Science Benchmarks. ${ }^{a}$ Site altitude to be greater than $4.4 \mathrm{~km}$ above sea level. ${ }^{b}$ Energy resolution $<O(30 \%)$ throughout core energy range $1-100 \mathrm{TeV} .{ }^{c}$ Angular resolution $\sim 0.15^{\circ}$ throughout core energy range 1-100 TeV. ${ }^{d}$ Site latitude not constraining among candidate sites under consideration. ${ }^{e} \mathrm{WCD}$ units with muon identification capability for $\gamma /$ hadron discrimnation.

\section{Science Benchmarks for SWGO}

The ultimate goal of a new observatory is to discover novel astrophysical phenomena that expand the frontiers of knowledge. Since these cannot be constrained beforehand, experimental design must proceed on the basis of reasonable extrapolations from current understanding (the known unknowns). To this purpose, a set of core science cases has been defined, as detailed in [9], which aim to guide the definition of options for the R\&D studies, and later to benchmark the final observatory design. They reflect the minimum set of science goals aimed for by the Observatory, and directly translate into detector performance requirements.

Table 1 briefly lists the core science goals discussed in Section 2, and their associated design characteristics, which imply a number of basic performance requirements: (i) a dense array core and excellent $\gamma$ /hadron separation for low-energy detection threshold, $<300 \mathrm{GeV}$; (ii) an extended sparse array with peak point source sensitivity at $\sim 100 \mathrm{TeV}$; (iii) muon tagging capability at WCD units for cosmic-ray studies and improved background suppression; and (iv) improved angular $\left(\sim 0.15^{\circ}\right)$ and energy $(<30 \%)$ resolutions throughout the core energy range $1-100 \mathrm{TeV}$. A set of quantitative benchmarks were derived which will be used to assess and compare the performance of the different SWGO candidate array configurations under investigation during the R\&D Phase. 


\section{Conclusions and Outlook}

Since the first VHE gamma-ray detection [26], hundreds of sources have been discovered, including variable objects. Their study requires instruments able to continuously monitor large portions of the sky, sensitive to energies above those of satellite-based experiments. The recent detection of photons of extreme energies from the Galaxy [6,27] strengthens the necessity for a large survey instrument with ample access to the Galactic Plane and sensitivity beyond $100 \mathrm{TeV}$. The vision pursued for SWGO [14] is of an observatory that aims to cover a wide energy range, bridging between satellite observations down to $100 \mathrm{GeV}$, and reaching towards the PeVs. Its location at high altitude in the Southern Hemisphere will provide a window to an unexplored sector of the sky and, particularly, to the centre of the Galaxy. Currently in R\&D Phase, the project plans to deliver an Observatory proposal by 2023.

\section{Acknowledgements}

The SWGO Collaboration acknowledges the support from the agencies and organizations listed here: https://wWW. swgo.org/SWGOWiki/doku . php?id=acknowledgements.

\section{References}

[1] B.P. Abbott, et al. Multi-messenger observations of a binary neutron star merger. Astrophys. J. Lett. 848:L12, 2017a.

[2] B.P. Abbott, et al. Gravitational Waves and Gamma-Rays from a Binary Neutron Star Merger: GW170817 and GRB 170817A. Astrophys. J. Lett. 848:L13, $2017 \mathrm{~b}$.

[3] M. Ackermann, et al. (The Fermi-LAT and Fermi-GBM Collaborations). Fermi-LAT Observations of the Gamma-Ray Burst GRB 130427A. Science 343:42-47, 2013.

[4] M. Ajello et al. A Decade of Gamma-Ray Bursts Observed by Fermi-LAT: The Second GRB Catalog. Astrophysical Journal 878:52, 2019.

[5] A. Albert, R. Alfaro, H. Ashkar, et al. Science Case for a Wide Field-of-View Very-High-Energy Gamma-Ray Observatory in the Southern Hemisphere, 2019, eprint arXiv: 1902.08429.

[6] M. Amenomori, et al. (Tibet $\mathrm{AS}_{\gamma}$ Collaboration) First Detection of sub-PeV Diffuse Gamma Rays from the Galactic Disk. Phys. Rev. Lett. 126:141101, 2021.

[7] E.O. Angüner, F. Cassol, H. Costantini et al. CherenkovTelescope Array potential in the search for Galactic PeVatrons, 2019, eprint arXiv: 1911.0613.

[8] X. Bai, et al. (LHAASO Collaboration) The Large High Altitude Air Shower Observatory (LHAASO) Science White Paper, 2019, eprint arXiv: 1905.02773.

[9] U. Barres de Almeida, et al. (SWGO Collaboration) The Southern Wide-Field Gamma-ray Observatory. Astron. Nachr. 342:431-437, 2021. 
[10] R. Conceição, B.S. González, A. Guillén, et al. Muon identification in a compact single-layered water Cherenkov detector and $\gamma /$ hadron discrimination, 2021, eprint arXiv:2101.10109.

[11] M. Doro et al. (The SWGO Collaboration) The search for high altitude sites in South America for the SWGO detector, PoS(ICRC2021)689, 2021.

[12] Z. Hampel-Arias and S. Westerhoff Gamma Hadron Separation using Pairwise Compactness Method with HAWC, 2016, eprint arXiv: 1508.04047.

[13] The HAWC Collaboration and The IceCube Collaboration Combined Analysis of Cosmic-Ray Anisotropy with IceCube and HAWC, 2017, eprint arXiv: 1708.03005.

[14] J. Hinton et al. (The SWGO Collaboration) The Southern Wide-field Gamma-ray Observatory: Status and Prospects, PoS(ICRC2021)023, 2021.

[15] The IceCube Collaboration, et al. Multimessenger observations of a flaring blazar coincident with high-energy neutrino IceCube-170922A. Science 361:1378, 2018.

[16] J. Knödlseder. The Cherenkov Telescope Array, 2020, eprint arXiv:2004.09213.

[17] G. La Mura, G. Chiaro, R. Conceição, et al. Detection of very-high-energy gamma-ray transients with monitoring facilities, MNRAS 497:3142-3148 (2020).

[18] G. La Mura et al. (The SWGO Collaboration) Monitoring Gamma-Ray Burst VHE emission with the Southern Wide-field-of-view Gamma-ray Observatory, PoS(ICRC2021)709, 2021.

[19] R. Lopez-Coto, A. Mitchell, E.O. Angüner et al. Galactic Science with the Southern Wide-field Gamma-ray Observatory, PoS(ICRC2021)892, 2021.

[20] MAGIC Collaboration, V. A. Acciari et al., Teraelectronvolt emission from the gamma-ray burst GRB 190114C. Nature 575:455-458, 2019.

[21] P. Montini, et al. (ARGO-YBJ Collaboration) Cosmic ray physics with ARGO-YBJ. Nuclear and Particle Physics Proceedings 279-281:7-14, 2016.

[22] A. Neronov and D. Semikoz. Lhaaso telescope sensitivity to diffuse gamma-ray signals from the galaxy. Phys. Rev. D 102:7-043025, 2020.

[23] J.D. Simon The Faintest Dwarf Galaxies. Ann. Rev. Astron. \& Astroph. 57:375-415, 2019.

[24] W. Springer, et al. (HAWC Collaboration) The High Altitude water Cherenkov (HAWC) Observatory. Nuclear and Particle Physics Proceedings 279-281:87-94, 2016.

[25] A. Viana et al. (The SWGO Collaboration). Searching for Dark Matter with the Southern Wide-field Gamma-ray Observatory (SWGO), PoS(ICRC2021)555, 2021.

[26] T.C. Weekes, M.F. Cawley, D.J. Fegan, et al. Observation of TeV Gamma Rays from the Crab Nebula Using the Atmospheric Cerenkov Imaging Technique. Astrophysical J., 342:379, 1989.

[27] C. Zhen, et al. (LHAASO Collaboration) Ultrahigh-energy photons up to $1.4 \mathrm{PeV}$ from 12 $\gamma$-ray Galactic sources. Nature, 594:33-36, 2021. 


\section{Full Authors List: SWGO Collaboration}

P. Abreu ${ }^{1}$, A. Albert ${ }^{2}$, E. O. Angüner ${ }^{3}$, C. Arcaro $^{4}$, L.H. Arnaldi ${ }^{5}$, J.C. Arteaga-Velázquez ${ }^{6}$, P. Assis ${ }^{1}$, A. Bakalová ${ }^{7}$, U. Barres de Almeida ${ }^{8}$, I. Batković ${ }^{4}$, J. Bellido ${ }^{9}$, E. Belmont-Moreno ${ }^{10}$, F. Bisconti ${ }^{11}$, A. Blanco ${ }^{1}$, M. Bohacova ${ }^{7}$, E. Bottacini ${ }^{4}$, T. Bretz ${ }^{12}$, C. Brisbois ${ }^{13}$, P. Brogueira ${ }^{1}$, A.M. Brown ${ }^{14}$, T. Bulik ${ }^{15}$, K. S. Caballero Mora ${ }^{16}$, S. M. Campos ${ }^{17}$ A. Chiavassa ${ }^{11}$, L. Chytka ${ }^{7}$, R. Conceição ${ }^{1}$, G. Consolati ${ }^{18}$, J. Cotzomi Paleta ${ }^{19}$, S. Dasso ${ }^{20}$, A. De Angelis ${ }^{4}$, C. R. De Bom ${ }^{8}$, E. de la Fuente ${ }^{21}$, V. de Souza ${ }^{22}$, D. Depaoli ${ }^{11}$, G. Di Sciascio ${ }^{23}$, C. O. Dib ${ }^{24}$, D. Dorner ${ }^{25}$, M. Doro ${ }^{4}$, M. Du Vernois ${ }^{26}$, T. Erginn $^{27}$, K. L. Fan ${ }^{13}$, N. Fraija ${ }^{8}$, S. Funk $^{28}$, J. I. García ${ }^{17}$, J. A. García-González ${ }^{29}$, S. T. García Roca ${ }^{9}$, G. Giacinti ${ }^{30}$, H. Goksu ${ }^{30}$, B. S. González ${ }^{1}$, F. Guarino ${ }^{31}$, A. Guillén ${ }^{32}$,

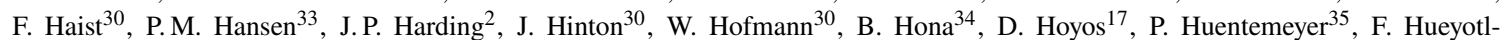
Zahuantitla $^{16}$ A. Insolia ${ }^{36}$, P. Janecek ${ }^{7}$, V. Joshi ${ }^{28}$, B. Khelifi ${ }^{37}$, S. Kunwar ${ }^{30}$, G. La Mura ${ }^{1}$, J. Lapington ${ }^{38}$, M. R. Laspiur ${ }^{17}$, F. Leitt ${ }^{28}$, F. Longo ${ }^{39}$, L. Lopes ${ }^{1}$, R. Lopez-Coto ${ }^{4}$, D. Mandat ${ }^{7}$, A. G. Mariazzi ${ }^{33}$, M. Mariotti ${ }^{4}$, A. Marques Moraes ${ }^{8}$, J. MartínezCastro $^{40}$, H. Martínez-Huerta ${ }^{41}$, S. May ${ }^{42}$, D. G. Melo ${ }^{43}$, L. F. Mendes ${ }^{1}$, L. M. Mendes ${ }^{1}$, T. Mineeva ${ }^{24}$, A. Mitchell ${ }^{44}$, S. Mohan ${ }^{35}$, O. G. Morales Olivares ${ }^{16}$, E. Moreno-Barbosa ${ }^{19}$, L. Nellen ${ }^{45}$, V. Novotny ${ }^{7}$, L. Olivera-Nieto ${ }^{30}$, E. Orlando ${ }^{39}$, M. Pech $^{7}$, A. Pichel $^{20}$, M. Pimenta ${ }^{1}$, M. Portes de Albuquerque ${ }^{8}$, E. Prandini ${ }^{4}$, M. S. Rado Cuchills ${ }^{9}$, A. Reisenegger ${ }^{46}$, B. Reville ${ }^{30}$, C. D. Rho ${ }^{47}$, A. C. Rovero ${ }^{20}$, E. Ruiz-Velasco ${ }^{30}$, G. A. Salazar ${ }^{17}$, A. Sandoval ${ }^{10}$, M. Santander ${ }^{42}$, H. Schoorlemmer ${ }^{30}$, F. Schüssler ${ }^{48}$, V. H. Serrano ${ }^{17}$, R. C. Shellard ${ }^{8}$, A. Sinha ${ }^{49}$, A. J. Smith ${ }^{13}$, P. Surajbali ${ }^{30}$, B. Tomé ${ }^{1}$, I. Torres Aguilar ${ }^{50}$, C. van Eldik $^{28}$, I. D. Vergara-Quispe ${ }^{33}$, A. Viana ${ }^{22}$, J. Vícha ${ }^{7}$, C. F. Vigorito ${ }^{11}$, X. Wang ${ }^{35}$, F. Werner ${ }^{30}$, R. White ${ }^{30}$, M. A. Zamalloa Jara ${ }^{9}$

${ }^{1}$ Laboratório de Instrumentação e Física Experimental de Partículas (LIP), Av. Prof. Gama Pinto 2, 1649-003 Lisboa, Portugal

2 Physics Division, Los Alamos National Laboratory, P.O. Box 1663, Los Alamos, NM 87545, United States

${ }^{3}$ Aix Marseille Univ, CNRS/IN2P3, CPPM, 163 avenue de Luminy - Case 902, 13288 Marseille cedex 09, France

${ }^{4}$ University of Padova, Department of Physics and Astronomy \& INFN Padova, Via Marzolo 8 - 35131 Padova, Italy

${ }^{5}$ Centro Atómico Bariloche, Comisión Nacional de Energía Atómica, S. C. de Bariloche (8400), RN, Argentina

${ }^{6}$ Universidad Michoacana de San Nicolás de Hidalgo, Calle de Santiago Tapia 403, Centro, 58000 Morelia, Mich., México

${ }^{7}$ FZU, Institute of Physics of the Czech Academy of Sciences, Na Slovance 1999/2, 18200 Praha 8, Czech Republic

${ }^{8}$ Centro Brasileiro de Pesquisas Físicas, R. Dr. Xavier Sigaud, 150 - Rio de Janeiro - RJ, 22290-180, Brazil

${ }^{9}$ Academic Department of Physics - Faculty of Sciences - Universidad Nacional de San Antonio Abad del Cusco (UNSAAC), Av. de la Cultura, 733, Pabellón C-358, Cusco, Peru

${ }^{10}$ Instituto de Física, Universidad Nacional Autónoma de México, Sendero Bicipuma, C.U., Coyoacán, 04510 Ciudad de México, CDMX, México

${ }^{11}$ Dipartimento di Fisica, Università degli Studi di Torino, Via Pietro Giuria 1, 10125, Torino, Italy

${ }^{12}$ RWTH Aachen University, Physics Institute 3, Otto-Blumenthal-Straße, 52074 Aachen, Germany

${ }^{13}$ University of Maryland, College Park, MD 20742, United States

${ }^{14}$ Durham University, Stockton Road, Durham, DH1 3LE, United Kingdom

${ }^{15}$ Astronomical Observatory, University of Warsaw, Aleje Ujazdowskie 4, 00478 Warsaw, Poland

${ }^{16}$ Facultad de Ciencias en Física y Matemáticas UNACH, Boulevard Belisario Domínguez, Km. 1081, Sin Número, Terán, Tuxtla Gutiérrez, Chiapas, México

${ }^{17}$ Facultad de Ciencias Exactas, Universidad Nacional de Salta, Avda. Bolivia No 5150, (4400) Salta Capital, Argentina

${ }^{18}$ Department of Aerospace Science and Technology, Politecnico di Milano, Via Privata Giuseppe La Masa, 34, 20156 Milano MI, Italy

${ }^{19}$ Facultad de Ciencias Físico Matemáticas, Benemérita Universidad Autónoma de Puebla, C.P. 72592, México

${ }^{20}$ Instituto de Astronomia y Fisica del Espacio (IAFE, CONICET-UBA), Casilla de Correo 67 - Suc. 28 (C1428ZAA), Ciudad

Autónoma de Buenos Aires, Argentina

${ }^{21}$ Universidad de Guadalajara, Blvd. Gral. Marcelino García Barragán 1421, Olímpica, 44430 Guadalajara, Jal., México

22 Instituto de Física de São Carlos, Universidade de São Paulo, Avenida Trabalhador São-carlense, no 400, Parque Arnold Schimidt CEP 13566-590, São Carlos - São Paulo - Brasil

${ }^{23}$ INFN - Roma Tor Vergata and INAF-IAPS, Via del Fosso del Cavaliere, 100, 00133 Roma RM, Italy

${ }^{24}$ Dept. of Physics and CCTVal, Universidad Tecnica Federico Santa Maria, Avenida España 1680, Valparaíso, Chile

${ }^{25}$ Universität Würzburg, Institut für Theoretische Physik und Astrophysik, Emil-Fischer-Str. 31, 97074 Würzburg, Germany

${ }^{26}$ Department of Physics, and the Wisconsin IceCube Particle Astrophysics Center (WIPAC), University of Wisconsin, 222 West Washington Ave., Suite 500, Madison, WI 53703, United States

27 TUBITAK Space Technologies Research Institute, ODTU Campus, 06800, Ankara, Turkey

${ }^{28}$ Friedrich-Alexander-Universität Erlangen-Nürnberg, Erlangen Centre for Astroparticle Physics, Erwin-Rommel-Str. 1, D 91058 Erlangen, Germany

${ }^{29}$ Tecnologico de Monterrey, Escuela de Ingeniería y Ciencias, Ave. Eugenio Garza Sada 2501, Monterrey, N.L., 64849, México

${ }^{30}$ Max-Planck-Institut für Kernphysik, P.O. Box 103980, D 69029 Heidelberg, Germany

${ }^{31}$ Università di Napoli "Federico II", Dipartimento di Fisica "Ettore Pancini”, and INFN Napoli, Complesso Universitario di Monte Sant'Angelo - Via Cinthia, 21 - 80126 - Napoli, Italy

${ }^{32}$ University of Granada, Campus Universitario de Cartuja, Calle Prof. Vicente Callao, 3, 18011 Granada, Spain 
${ }^{33}$ IFLP, Universidad Nacional de La Plata and CONICET, Diagonal 113, Casco Urbano, B1900 La Plata, Provincia de Buenos Aires, Argentina

${ }^{34}$ University of Utah, 201 Presidents' Cir, Salt Lake City, UT 84112, United States

${ }^{35}$ Michigan Technological University, 1400 Townsend Drive, Houghton, MI 49931, United States

${ }^{36}$ Dipartimento di Fisica e Astronomia "E. Majorana", Catania University and INFN, Catania, Italy

${ }^{37}$ APC-IN2P3/CNRS, Université de Paris, Bâtiment Condorcet, 10 rue A.Domon et Léonie Duquet, 75205 PARIS CEDEX 13, France

${ }^{38}$ University of Leicester, University Road, Leicester LE1 7RH, United Kingdom

${ }^{39}$ Department of Physics, University of Trieste and INFN Trieste, via Valerio 2, I-34127, Trieste, Italy

${ }^{40}$ Centro de Investigación en Computación, Instituto Politécnico Nacional, Av. Juan de Dios Bátiz S/N, Nueva Industrial Vallejo, Gustavo A. Madero, 07738 Ciudad de México, CDMX, México

${ }^{41}$ Department of Physics and Mathematics, Universidad de Monterrey, Av. Morones Prieto 4500, San Pedro Garza García 66238, N.L., México

42 Department of Physics and Astronomy, University of Alabama, Gallalee Hall, Tuscaloosa, AL 35401, United States

${ }^{43}$ Instituto de Tecnologías en Detección y Astropartículas (CNEA-CONICET-UNSAM), Av. Gral Paz 1499 - San Martín - Pcia. de Buenos Aires, Argentina

${ }^{44}$ Department of Physics, ETH Zurich, CH-8093 Zurich, Switzerland

${ }^{45}$ Instituto de Ciencias Nucleares, Universidad Nacional Autónoma de México (ICN-UNAM), Cto. Exterior S/N, C.U., Coyoacán, 04510 Ciudad de México, CDMX, México

${ }^{46}$ Departamento de Física, Facultad de Ciencias Básicas, Universidad Metropolitana de Ciencias de la Educación, Av. José Pedro Alessandri 774, Ñuñoa, Santiago, Chile

${ }^{47}$ Department of Physics, University of Seoul, 163 Seoulsiripdaero, Dongdaemun-gu, Seoul 02504, Republic of Korea

${ }^{48}$ Institut de recherche sur les lois fondamentales de l'Univers (IRFU), CEA, Université Paris-Saclay, F-91191 Gif-sur-Yvette, France

${ }^{49}$ Laboratoire Univers et Particules de Montpellier, CNRS, Université de Montpelleir, F-34090 Montpellier, France

${ }^{50}$ Instituto Nacional de Astrofísica, Óptica y Electrónica (INAOE), Luis Enrique Erro 1, Puebla, México 